\title{
Performances of 16QAM with Fading Compensation and Postdetection Diversity Reception in Satellite Mobile Channels
}

\author{
H K LAU \\ hklau@eee.hku.hk \\ S W CHEUNG* \\ swcheung@eee.hku.hk \\ Communications Research Group \\ Department of Electrical and Electronic Engineering \\ The University of Hong Kong \\ HONG KONG \\ Phone: (852) 2859-2425 \\ Fax: (852) 2559-8738
}

* Please send all correspondence to Dr S W Cheung

Keywords: $\quad$ 16QAM; Diversity Reception; Fading Compensation; Satellite Mobile Channels, Data-Aided Technique

\begin{abstract}
The paper studies the effects of $N$-branch postdetection selection diversity reception, where $N=1,2,3$ or 4 , incorporated with fading compensation on a digital satellite mobile system. The digital satellite mobile system transmits a pilot-symbol-aided 16-ary quadratureamplitude modulated (PSA-16QAM) signal over the Rician channels. A selection method that makes use of the pilot symbols to select one of the $N$ branches in the diversity reception system for signal detection and a novel PSA technique that makes use of both the pilot symbols and data symbols for fading compensation are proposed. Computer simulation tests are used to assess the effects of the proposed techniques on the bit-error rate performances (BER) of the PSA-16QAM system in the presence of additive white Gaussian noise (AWGN) or co-channel interference (CCI) in the Rician faded channels.

When frequency diversity is used, PSA-16QAM with 2-branch and 4-branch diversity reception occupies about the same bandwidths as quaternary phase-shift-keying (QPSK) without using diversity and with 2-branch diversity, respectively, yet achieving the same capacity. Thus simulation tests on the BER performances of a QPSK system without diversity and with 2-branch diversity are also carried and the results are used to determine the preferred system arrangements.
\end{abstract}




\section{INTRODUCTION}

The conventional modulation scheme for digital satellite mobile communications systems has been quaternary phase-shift keying (QPSK) with a theoretical spectral efficiency of only 2 bit/s/Hz. With mobile communications systems migrating to global wireless personal communications systems, the demands for channel capacity in digital satellite mobile systems are likely to be tremendous [1-2]. Signals with higher spectrally efficiencies than QPSK therefore need to be considered for future use in digital satellite mobile systems. Among these signals, the 16-ary quadrature-amplitude modulation (16QAM) is one of the promising candidates because of its relative simplicity of implementation and good error-rate performance through linear channels. Studies have been carried out on the bit-error rate (BER) performance of 16QAM in the satellite mobile channels and results have shown that the fading distortion causes very high BER floors to the signal [3-4].

Pilot-symbol-aided (PSA) transmission has been proposed for transmitting spectrally efficient signals such as 16QAM over the fading channels. In a PSA system, the transmitted data symbol sequence is divided into frames of symbols for transmission. A known pilot symbol is inserted at the beginning of each frame. At the receiver, these pilot symbols are extracted from the received signal and used to estimate the signal distortion introduced in the fading channel. The resultant estimates are then used to correct the fading effects of the data symbols. Several methods have been proposed in estimating the fading effects [5-9]. In these studies, the receivers make use of only the pilot symbols and ignore the fading information in the data symbols. A PSA technique that uses both the pilot symbols and data symbols in zeroth-order estimation to enhance the accuracy of the estimation process has been proposed [4]. Although results have shown that substantial improvements can be obtained by using this estimation method, the required signal-to-noise ratios to achieve a practical error rate of $10^{-3}$ are quite high [4]. An improved estimation technique that uses both the pilot symbols and data symbols in first-order estimation has also been proposed, and the results have shown that, in the Rayleigh fading channels, substantial improvements on the BER performances can be achieved [10]. 
Diversity reception is one of the effective techniques for combating multipath fading [11-14]. For mobile systems, postdetection diversity is more attractive because the cophasing function in the predetection combiners is not required [12]. In this paper, the BER performances of a PSA-16QAM signal incorporated with postdetection diversity reception for use in a satellite mobile system are studied. The satellite channel is Rician faded. The system employs $N$-branch postdetection selection diversity reception where $N=1,2,3$ or 4 . A selection method that makes use of the pilot symbols to select one of the $N$ branches in the diversity reception system for signal detection and a novel PSA technique that makes use both the pilot symbols and the data symbols in first-order estimation to enhance the accuracy of the estimation process of the fading channel are proposed. A series of computer simulation tests has been carried out to assess the effects of the proposed techniques on the BER performances of the PSA-16QAM system, in the presence of additive white Gaussian noise (AWGN) or co-channel interference (CCI) in the Rician channels. In all tests, it is assumed the direct components in all the transmission paths have the same CCI power, the system is operating at a rate of $32 \mathrm{~kb} / \mathrm{s}$ or 8 kbauds with a signal carrier frequency of $1.8 \mathrm{GHz}$, and the radial velocity between the transmitter and receiver is $48 \mathrm{~km} / \mathrm{hr}$, resulting in a normalized Doppler spread of 0.01 .

An advantage of using frequency diversity is that the number of receiving antennas can be reduced to one, with the disadvantage that a wider bandwidth is required. If frequency diversity is used, PSA-16QAM with 2-branch and 4-branch diversities occupies about the same bandwidths as QPSK without using diversity and with 2-branch diversity, respectively, yet achieving the same capacity. Thus simulation tests on the BER performances of a QPSK system without using diversity and with 2-branch diversity have also been carried out. 


\section{SYSTEM MODEL}

The baseband equivalent data-transmission model used in the study is shown in Fig. 1. When the encoder has received the binary information $\left\{u_{n}\right\}$ at time $t=n T$ seconds (where $n$ is a positive integer and $T$ is the symbol duration), it maps these signals into the appropriate data symbols $\left\{d_{n}\right\}$ according to the square 16QAM signal constellations. For every ( $L-1)$-data symbols, a pilot symbol from a known pseudorandom-symbol sequence $\left\{p_{n}\right\}$ is inserted to form a frame of $L$-symbol long as shown in Fig. 2. A pseudorandom sequence of pilot symbols is used to avoid transmitting tones [5]. To minimize the performance degradation due to AWGN, the $\left\{p_{n}\right\}$ are chosen from those signal vectors with the largest energy levels in the signal constellations [8]. It is assumed that the receiver has the prior knowledge of $\left\{p_{n}\right\}$. In Fig. 1, at time $t=n T$ seconds, the (data or pilot) symbol to be transmitted is used to form the impulse $q_{n} \delta(t-n T)$, which is fed to the premodulation filter with an impulse response $a(t)$. The $q_{n}$ is complex-valued and is either a data symbol or a pilot symbol, and $\delta(t)$ is the Dirac delta function. At the output of the premodulation filter, the signal becomes $\sum_{n} q_{n} a(t-n T)$ which is then used to linearly modulate a carrier signal to produce the transmit signal.

Each of the transmission paths (transmission paths $\# 1, \# 2, \ldots$, and $\# N$ ) in Fig. 1 is a land mobile satellite channel that introduces Rician fading distortion to the corresponding input signal. The Rician-faded signal is composed of a direct component and a multipath component as shown in Fig. 3a. The multipath component is assumed to be Rayleigh-faded and is generated by multiplying the direct component by a complex-valued parameter $h(t)$ to give $\sum_{n} q_{n} a(t-n T) h(t)$ as shown in Fig. 3b [15]. The two lowpass filters in Fig. 3b are two second-order Butterworth filters with the same normalized power-spectral-density of Mason [16]

$$
B(f)=\frac{1}{1+\left(f / f_{D}\right)^{4}}
$$

The quantity $f_{D}$ determines the maximum Doppler frequency of the multipath component and is given by Salwch [17] 


$$
f_{D}=\frac{v}{\lambda}
$$

where $v$ is the radial velocity of the mobile and $\lambda$ is the wavelength of the signal carrier. In Fig. 3b, the two amplifiers with a gain of $K$ are used to adjust the power ratio of the directcomponent to the multipath-component in the simulation tests. Each transmission path is called a diversity branch. The signals in these branches are statistically uncorrelated and independent with each others. In the study, the direct components in these branches are assumed to have the same power level.

The $N$ receivers in Fig. 1 account for an $N$-branch diversity reception system. Both AWGN and CCI are assumed to be added at the inputs of the $N$ receivers. The CCI in each of the branches is generated by using a separate transmitter, thus the noise and CCI in these branches are statistically uncorrelated and indepedent with each others. The selection circuit selects a data frame from an output of these $N$ receivers for signal detection. The proposed selection method is described in the next section.

Assume that the signal from receiver $\# M$ has been selected for signal detection. The baseband equivalent signal from receiver \#M is given by

$$
\begin{aligned}
& \sum_{n} q_{n} a(t-n T)+K \sum_{n} q_{n} a(t-n T) h_{M}(t)+v_{M}(t)+c_{M}^{*}(t) \\
& =\sum_{n}\left[q_{n} a(t-n T) y_{M}(t)\right]+v_{M}(t)+c_{M}^{*}(t)
\end{aligned}
$$

where $y_{M}(t)=1+K h_{M}(t)$ is the signal distortion introduced in transmission path \#M, $v_{M}(t)$ is the AWGN waveform with a one-sided power spectrum of $N_{0}$, and $c_{M}^{*}(t)$ is the CCI waveform. The received signal is filtered by a postdetection filter which is taken to have the same impulse response $a(t)$ as the premodulation filter in the transmitter. The resultant transfer function of the premodulation filter and the postdetection filter in cascade has a sinusoidal roll-off of $100 \%$. At the output of the postdetection filter, the baseband signal is

$$
r_{M}(t)=\sum_{n}\left[q_{n} a(t-n T) y_{M}(t)\right] * a(t)+w_{M}(t)+c_{M}(t)
$$


where $*$ denotes the convolution process, $w_{M}(t)=v_{M}(t) * a(t)$ and $c_{M}(t)=c_{M}^{*}(t) * a(t)$ are the filtered noise and CCI waveforms, respectively, at receiver \#M.

In the mobile satellite systems, the Doppler shift of the received signal caused by the motion of the mobile is small compared to the symbol rate, so that the inter-symbolinterference (ISI) introduced by the filtering process can be neglected. The baseband signal $r(t)$ is sampled in synchronism at the time instants $\{n T\}$. For convenience, it is assumed that $a(t) * a(t)=1$ at time $t=0$. Therefore, the signals at time $t=n T$ seconds is

$$
r_{M, n}=q_{n} y_{M, n}+w_{M, n}+c_{M, n}
$$

where $y_{M, n}=y_{M}(n T), w_{M, n}=w_{M}(n T)$ and $c_{M, n}=c_{M}(n T)$. If $q_{n}$ is a pilot symbol and is known at the receiver, in the absence of noise and CCI, the signal $y_{M, n}$ can be obtained as

$$
y_{M, n}=\frac{r_{M, n}}{q_{n}}
$$

However, in the presence of noise and/or CCI, only an estimate of $y_{M, n}$ can be obtained. The estimate, $\hat{y}_{M, n}$, is then used for fading compensation (estimation and correction) of the received data symbols in the associated frame. The corrected data symbols are fed to the detector and decoder to produce the binary data $\left\{\hat{u}_{n}\right\}$ at the output. The proposed compensation technique is described in the following section. 


\section{SELECTION METHOD AND FADING COMPENSATION TECHNIQUE}

Fading Esimation Using Pilot Symbols

It is assumed here that frame synchronization of the received signals from the $N$ receivers has been achieved. At the output of the postdetection filter in receiver \#J, where $J=$ $1,2, \ldots$, or $N$, the sample signal at the $i$-th position of the $k$-th received frame can be written as

$$
r_{J, k, i}=q_{k, i} y_{J, k, i}+w_{J, k, i}+c_{J, k, i}
$$

where $q_{k, i}$ is either a pilot symbol or a data symbol, $y_{J, k, i}$ is the change due to fading distortion, $w_{J, k, i}$ is the sample of AGN and $c_{J, k, i}$ is the sample of CCI. For $i=0$, the signal is

$$
r_{J, k, 0}=p_{k, 0} y_{J, k, 0}+w_{J, k, 0}+c_{J, k, 0}
$$

where $p_{k, 0}$ is the pilot symbol in the $k$-th frame. For $i=1,2, \ldots, L-1$, the signal is

$$
r_{J, k, i}=d_{k, i} y_{J, k, i}+w_{J, k, i}+c_{J, k, i}
$$

where $d_{k, i}$ is a data symbol at the $i$-th position of the $k$-th frame. Since the pilot symbol $p_{k, 0}$ is known at the receivers, $y_{J, k, 0}$ for receiver $\# J$ can be obtained, from Eqn. (8), as

$$
y_{J, k, 0}=\frac{r_{J, k, 0}}{p_{k, 0}}-\frac{w_{J, k, 0}}{p_{k, 0}}-\frac{c_{J, k, 0}}{p_{k, 0}}
$$

In a low noise and low CCI environment, $y_{J, k, 0}$ and $y_{J, k+1,0}$ can be estimated, respectively, as

$$
\begin{gathered}
\hat{y}_{J, k, 0}=\frac{r_{J, k, 0}}{p_{k, 0}} \\
\hat{y}_{J, k+1,0}=\frac{r_{J, k+1,0}}{p_{k+1,0}}
\end{gathered}
$$




\section{Selection Method}

There are $N$ uncorrelated faded signals from these diversity branches and so a method is proposed here to select one of them for signal detection. Since data errors are more likely resulted from the received signals in a deep fade (weak signal strength), the pilot symbols which are known at the receivers can be used as indications of the strengths of these $N$ received signals and hence to select the strongest one. Thus, for the signals in the $k$-th frames of these signals, the selection circuit computes the values of

$$
H_{J}=\left|\operatorname{Re}\left(\hat{y}_{J, k, 0}+\hat{y}_{J, k+1,0}\right)\right|+\left|\operatorname{Im}\left(\hat{y}_{J, k, 0}+\hat{y}_{J, k+1,0}\right)\right|
$$

for $J=1,2, \ldots$, and $N$, using the two pilot symbols in the associated $k$-th and $(k+1)$-th frames. The circuit then selects the $k$-th data frame associated with the largest value of $H_{J}$ from these $N$ receivers. If more than one receivers have the same value of $H_{J}$, the selection circuit selects any one of them. Once a received frame is selected for signal detection, the fading compensation process begins.

The compensation process proposed here is suitable for frame lengths of $4,8,16, \ldots$, or $2^{m}$, where $m$ is an integer, and consists of two compensation stages. The first stage applies to the data symbols in the even-number positions (i.e., $i=2,4, \ldots$, and $L$-2), while the second stage applies to the data symbols in the odd-number positions (i.e., $i=1,3, \ldots$, and $L-1$ ) of the selected frame.

\section{Compensation for Data Symbols in Even-Number Positions}

Here, assume that $H_{M}$ has the largest value and so the data frame from receiver \#M is selected for signal detection. The estimate of the distortion effects $y_{M, k, L / 2}$ on the data symbol $d_{k, L / 2}$ in the data frame is computed as

$$
\tilde{y}_{M, k, L / 2}=\frac{\hat{y}_{M, k, 0}+\hat{y}_{M, k+1,0}}{2}
$$


This signal is used to correct the fading effects on $r_{M, k, L / 2}$ to give an estimate of the data symbol

$$
\hat{r}_{M, k, L / 2}=\frac{r_{M, k, L / 2}}{\tilde{y}_{M, k, L / 2}}
$$

which is threshold detected to produce the data symbol $\hat{d}_{k, L / 2}$ (where $\hat{d}_{k, L / 2}$ is a possible signal vector on the signal constellation). If $L=4$, the compensation process for the data symbols in the even-number positions is completed and the compensation process for the data symbols in the odd-number positions begins.

If $L=8$, the compensation process for the data symbols in the even-number positions continues as follows. The detected data $\hat{d}_{k, L / 2}$ is used to estimate $y_{M, k, L / 2}$ as $r_{M, k, L / 2} / \hat{d}_{k, L / 2}$ which is then used to obtain the estimates of $y_{M, k, L / 4}$ and $y_{M, k, 3 L / 4}$ as

$$
\begin{gathered}
\tilde{y}_{M, k, L / 4}=\frac{1}{2}\left(\hat{y}_{M, k, 0}+\frac{r_{M, k, L / 2}}{\hat{d}_{k, L / 2}}\right) \\
\tilde{y}_{M, k, 3 L / 4}=\frac{1}{2}\left(\frac{r_{M, k, L / 2}}{\hat{d}_{k, L / 2}}+\hat{y}_{M, k+1,0}\right)
\end{gathered}
$$

respectively. These signals $\tilde{y}_{M, k, L / 4}$ and $\tilde{y}_{M, k, 3 L / 4}$ are used to correct $r_{M, k, L / 4}$ and $r_{M, k, 3 L / 4}$ to obtain the data symbols

$$
\begin{gathered}
\hat{r}_{M, k, L / 4}=\frac{r_{M, k, L / 4}}{\tilde{y}_{M, k, L / 4}} \\
\hat{r}_{M, k, 3 L / 4}=\frac{r_{M, k, 3 L / 4}}{\tilde{y}_{M, k, 3 L / 4}}
\end{gathered}
$$

which are threshold detected to produce the corresponding data symbols $\hat{d}_{k, L / 4}$ and $\hat{d}_{k, 3 L / 4}$.

If $L=16$, the compensation process continues further. The detected data symbols, $\hat{d}_{k, L / 4}$ and $\hat{d}_{k, 3 L / 4}$, are used to obtained the estimates of $y_{M, k, L / 4}$ and $y_{M, k, 3 L / 4}$ as $r_{M, k, L / 4} / \hat{d}_{k, L / 4}$ and $r_{M, k, 3 L / 4} / \hat{d}_{k, 3 L / 4}$, respectively. Then together with $\hat{y}_{M, k, 0}$ and $\hat{y}_{M, k+1,0}$, the estimates of the effects on the rest of the symbols within the frame are obtained according to 


$$
\begin{gathered}
\tilde{y}_{M, k, L / 8}=\frac{1}{2}\left(\hat{y}_{M, k, 0}+\frac{r_{M, k, L / 4}}{\hat{d}_{k, L / 4}}\right) \\
\tilde{y}_{M, k, 7 L / 8}=\frac{1}{2}\left(\frac{r_{M, k, 3 L / 4}}{\hat{d}_{k, 3 L / 4}}+\hat{y}_{M, k+1,0}\right) \\
\tilde{y}_{M, k, j L / 8}=\frac{1}{2}\left(\frac{r_{M, k,(j-1) L / 8}}{\hat{d}_{k,(j-1) L / 8}}+\frac{r_{M, k,(j+1) L / 8}}{\hat{d}_{k,(j+1) L / 8}}\right) \text { for } j=3,5
\end{gathered}
$$

These estimates are used to correct the corresponding received data symbols. Here again, the corrected data symbols are then fed to the threshold detector to produce the detected data symbols. A set of equations similar to Eqn. (15) can be derived for $L=32,64, \ldots, 2^{m}$, where $m$ is an integer. The compensation process continues until all the data symbols in the even-number positions are done. Then the compensation process for the data symbols in the odd-number positions begins.

\section{Compensation for Data Symbols in Odd-Number Positions}

The compensation process for the data symbols in the odd-number positions is quite straightforward. The distortion effects on the data symbols are estimated as

$$
\begin{gathered}
\tilde{y}_{M, k, 1}=\frac{1}{2}\left(\hat{y}_{M, k, 0}+\frac{r_{M, k, 2}}{\hat{d}_{k, 2}}\right) \\
\tilde{y}_{M, k, L-1}=\frac{1}{2}\left(\frac{r_{M, k, L-2}}{\hat{d}_{k, L-2}}+\hat{y}_{M, k+1,0}\right) \\
\tilde{y}_{M, k, j}=\frac{1}{2}\left(\frac{r_{M, k, j-1}}{\hat{d}_{k, j-1}}+\frac{r_{M, k, j+1}}{\hat{d}_{k, j+1}}\right) \text { for } j=3,5, \ldots, L-3
\end{gathered}
$$

The corrected data symbols are then obtained as

$$
\hat{r}_{M, k, i}=\frac{r_{M, k, i}}{\tilde{y}_{M, k, i}} \quad \text { for } i=1,3, \ldots, L-1
$$


which are fed to the threshold detector to produce the detected data symbols. Of course, at the end of the compensation process, all the detected data symbols are fed to the decoder to produce the binary data $\left\{\hat{u}_{n}\right\}$.

Every time, when a frame is selected by the selection circuit from these $N$ receivers, the whole compensation process repeats. The major advantages of the proposed estimation technique are simple implementation and the short storage-delay time introduced by the fading compensation process. Since it requires only two pilot symbols to compensate all the data symbols within a frame, the storage-delay time is only one frame long.

\section{Signal Energy and Bandwidth Considerations}

Since the pilot symbols are known to the receiver, they carry no information, but require a certain amount of power and bandwidth for transmission. Thus for a system with a fixed transmission power, a portion of the power has to be assigned for transmitting the pilot symbols. The net average data-symbol energy is therefore reduced. Similarly, if the same system throughput as that without transmitting the pilot symbols is to be maintained, the resultant symbol rate needs to be reduced. These have been considered somewhere else [4]. 


\section{COMPUTER SIMULATION TESTS}

Basic assumptions

A series of computer-simulation tests has been carried out to investigate the BER performances of a 16QAM signal in the $N$-branch postdetection selection diversity reception system of Fig. 1. In every case the equivalent baseband model of the system in the time domain has been used and, for every symbol duration, a total of 8 equally spaced samples are taken to prevent aliasing, thus the filtering operations are carried out by convolution of the sampled signals and the sampled impulse responses of the filters. The simulation tests employ the Monte-Carlo method [18]. The detailed description of the simulation method has been discussed elsewhere [19]. Each individual measurement of the BER, at the given signalto-noise ratio, has been made with the transmission of enough data symbols to cause more than 500 error events. As a results, the $99 \%$ confidence limits are within $\pm 10 \%$ of the true BER [20]. In all tests, the direct components in all the transmission paths are assumed to have the same power and all the transmission paths from the same CCI power. A normalized Doppler spread of 0.01 (correspond to a transmission rate of $32 \mathrm{kbit} / \mathrm{s}$ at a radial velocity of $48 \mathrm{~km} / \mathrm{hr}$ and carrier frequency of $1.8 \mathrm{GHz}$ ), a frame length of $L=8$ and $N=1,2,3$, and 4 have been used. The signal-to-noise ratio is taken as

$$
\mathrm{SNR}=10 \log \left(\frac{E_{b}}{N_{0}}\right)
$$

where $E_{b}$ is the average bit energy (after taking into account the pilot symbols) and $N_{0}$ is the one-sided power spectral density of the AWGN.

In Fig. 3b, the two amplifiers with a gain of $K$ are used to adjust the power ratio of the direct-component to the multipath-component in the simulation tests. This ratio is denoted as

$$
\operatorname{SMR}=10\left[\log \left(\frac{S}{M}\right)\right]
$$

where $S$ is the direct-component power and $M$ is the multipath-component power in the transmission path. 
The $N$ receivers in Fig. 1 account for an $N$-branch diversity reception system. Both AWGN and CCI are assumed to be added at the inputs of the $N$ receivers. The signal-to-CCI power ratio is defined as

$$
\mathrm{SIR}=10\left[\log \left(\frac{S}{I}\right)\right]
$$

where $I$ is the power of CCI.

\section{Results and discussions}

In the absence of CCI, with SMR $=0 \mathrm{~dB}$ and $7 \mathrm{~dB}$, the BER performances of the 16QAM system as function of SNRs are shown in Figs. 4a and 4b, respectively. Note that $\mathrm{SMR}=0 \mathrm{~dB}$ means that the multipath component has the same power as the direct component and $N=1$ implies that the system does not use any diversity reception. It can be seen that diversity reception can significantly improve the BER performances of system. The improvement is more significant at the lower BERs. Furthermore, more improvements can be obtained by using more branches for reception, as is expected. Figure 4a shows that, without diversity reception and $\mathrm{SMR}=0 \mathrm{~dB}$, the system requires a SNR of about $16 \mathrm{~dB}$ to achieve a BER of $10^{-2}$. However, with 2-branch diversity reception, i.e., $N=2$, the system achieves the same BER at a SNR of only $10 \mathrm{~dB}$, gaining an advantage of $6 \mathrm{~dB}$ in the SNR. If $N=3$ (i.e., 3branch diversity reception) is used, the required SNR is further reduced to about $8 \mathrm{~dB}$. At a lower BER of $10^{-3}$, Fig. 4a shows that, without the use of diversity reception, the system requires a SNR of about $30 \mathrm{~dB}$. While, with $N=2$, the system can achieve this BER at a SNR of only about $16 \mathrm{~dB}$ which is further reduced to about $12.5 \mathrm{~dB}$ if 3-branch diversity reception is used. Thus the additional advantages of 14 and $17.5 \mathrm{~dB}$ can be obtained by using 2-branch and 3-branch diversity reception, respectively. These additional advantages are larger at the even lower BERs. If 4-branch diversity reception is used, then the further advantages at the BERs of $10^{-2}$ and $10^{-3}$ are 1 and $1.5 \mathrm{~dB}$, respectively, relative to those of 3-branch diversity reception. 
Figure 4b shows the BER performances of the PSA-16QAM system, in the absence of CCI, with SMR $=7 \mathrm{~dB}$ which is specified in the INMARSAT systems specifications [15]. Without diversity reception, the system requires a SNR of $13 \mathrm{~dB}$ to achieve a BER of $10^{-2}$; while with 2-branch diversity reception, the required SNR is $10.5 \mathrm{~dB}$, gaining an advantage of about $2.5 \mathrm{~dB}$. At a lower BER of $10^{-3}$, the 2-branch diversity reception system requires a SNR of $14.5 \mathrm{~dB}$; whereas without diversity reception, the system requires a SNR of $19.5 \mathrm{~dB}$. Here an advantage of about $4.5 \mathrm{~dB}$ is achieved by using 2-branch diversity reception. The advantage of using 3-branch diversity reception is only significant at the lower BERs. Fig 4b shows that, at the BERs tested, the additional advantages by using 4-branch diversity reception, instead of 3-branch diversity reception, is less than $1 \mathrm{~dB}$. Comparing Fig. 4a to Fig. $4 \mathrm{~b}$ shows that the advantages obtained by using the proposed $N$-branch diversity reception method are more significant with $\mathrm{SMR}=0 \mathrm{~dB}$ than with $\mathrm{SMR}=7 \mathrm{~dB}$.

The simulation results on the BER performances of a QPSK system with frequency diversity reception ( $N=1$ and 2) and ideal coherent detection are also shown in Fig. 4. (Note that PSA-16QAM with 2-branch and 4-branch frequency diversities occupies about the same bandwidths as QPSK without using diversity and with 2-branch diversity, respectively, yet having the same capacity.) In these tests, it is assumed that the coherent bandwidth of the signals are small, so that the signals received from different diversity branches are uncorrelated. Of course, if the coherent bandwidth is large, then the signals received from the diversity branches are highly correlated and frequency diversity reception will be less effective. It can be seen in Fig. 4 that the 2-branch diversity PSA-16QAM system outperforms the non-diversity QPSK system at the low BERs. With SMR $=0 \mathrm{~dB}$ and $7 \mathrm{~dB}$, the 2-branch diversity PSA-16QAM system starts gaining the advantages over the QPSK system at the BERs of below $8 \times 10^{-3}$ and $4 \times 10^{-4}$, respectively. The advantage is larger at the lower BERs. At a BER of $10^{-4}$, the 2-branch diversity PSA-16QAM system gains the advantages of $7 \mathrm{~dB}$ (from $28.5 \mathrm{~dB}$ to $21.5 \mathrm{~dB}$ ) and $2 \mathrm{~dB}$ (from $20 \mathrm{~dB}$ to $18 \mathrm{~dB}$ ), at SMR $=0 \mathrm{~dB}$ and $7 \mathrm{~dB}$, respectively, over the non diversity QPSK system. Thus if the system is designed to operate at low BERs, PSA-16QAM with 2-branch diversity is more preferred than non diversity QPSK. With SMR $=0 \mathrm{~dB}$, Fig. 4a shows that PSA-16QAM with $N=4$ 
gains advantages over QPSK with $N=2$ only at the BERs of below $2 \times 10^{-5}$. Thus PSA16QAM with 4-branch diversity is better than QPSK with 2-branch diversity if low BERs are desired. With SMR $=7 \mathrm{~dB}$, Fig. 4b shows that PSA-16QAM with 4-branch diversity is inferior to QPSK with 2-branch diversity in all the conditions tested. Thus at high SMRs, 2branch diversity QPSK is always better in BER performances than 4-branch diversity PSA16QAM, yet having the same capacity. Table 1 summarizes the required SNRs for $N$-branch diversity reception to achieve the BERs of $10^{-2}, 10^{-3}, 10^{-4}$ and $10^{-5}$.

In the absence of noise, the BER performances of the PSA-16QAM system in the CCI environments are shown in Fig. 5. With the multipath component having the same power as the direct component, i.e., SMR $=0 \mathrm{~dB}$, and no diversity reception, Fig. 5a shows that the system achieves a BER of $10^{-2}$ at a SIR of about $21.5 \mathrm{~dB}$. When 2-branch diversity reception is used, the required SIR reduces to $15.5 \mathrm{~dB}$, leading to an advantage of $6 \mathrm{~dB}$ in SIR over the non-diversity system. The advantage again is larger at a lower BER. Under the conditions tested, the non-diversity system cannot achieve the BER of $10^{-3}$; while with 2-branch diversity reception, the system achieves the BER of $10^{-3}$ at a SIR of about $21.5 \mathrm{~dB}$. An additional advantage of $1.5 \mathrm{~dB}$ in SIR can be obtained by 3-branch diversity reception. The further improvement by using 4-branch diversity reception, instead of 3-branch, is less significant. With $\mathrm{SMR}=7 \mathrm{~dB}$, the results are shown in Fig. $5 \mathrm{~b}$ which indicates that at the BERs of $10^{-2}$ and $10^{-3}$, 2-branch diversity reception provides the advantages of only about $2.5 \mathrm{~dB}$ (from $19 \mathrm{~dB}$ to $16.5 \mathrm{~dB}$ ) and $4 \mathrm{~dB}$ (from $25.5 \mathrm{~dB}$ to $21.5 \mathrm{~dB}$ ), respectively, over the non-diversity system. The further advantages obtained by using 3- or 4-branch diversity reception are quite insignificant. Once again, the BER performances of QPSK in the CCI environments, with $N=1$ and 2, and ideal coherent detection, are also shown in Fig. 5. It can be seen that, with SMR $=0 \mathrm{~dB}$ and $7 \mathrm{~dB}$, the PSA-16QAM system with 2-branch diversity reception starts gaining advantages over the non diversity QPSK system at the BERs of $2 \times 10^{-3}$ and $5 \times 10^{-5}$, respectively. However, in all the conditions tested, the 4-branch diversity PSK-16QAM system is inferior to the 2-branch diversity QPSK system and so is not preferred. Table 2 summarizes the required SIRs for $N$-branch diversity reception to achieve the BERs of $10^{-2}, 10^{-3}, 10^{-4}$ and $10^{-5}$. 


\section{CONCLUSIONS}

The effects of the proposed diversity reception techniques on the BER performances of a PSA-16QAM system incorporated with $N$-branch postdetection selection diversity reception in the satellite mobile environments have been studied. Results have shown that the tolerances to AWGN, CCI are significantly increased by the proposed techniques.

With 2-branch diversity reception and in the absence of CCI, at SMR $=0 \mathrm{~dB}$, PSA16QAM gains an advantage of $6 \mathrm{~dB}$ in the SNR at a BER of $10^{-2}$, over the case without using diversity reception. This advantage increases to $14 \mathrm{~dB}$ at a BER of $10^{-3}$. More advantages can be obtained by using 3-branch diversity reception. Under the same assumed conditions but at $\mathrm{SMR}=7 \mathrm{~dB}$, the corresponding advantages become $2.5 \mathrm{~dB}$ and $4.5 \mathrm{~dB}$, respectively. The advantages obtained by using more branches are relatively small.

With 2-branch diversity reception and in the absence of noise, at SMR $=0 \mathrm{~dB}$, the system gains an advantage of $6 \mathrm{~dB}$ in the SIR at a BER of $10^{-2}$, over the case without using diversity reception. At $\mathrm{SMR}=7 \mathrm{~dB}$, the advantage become $2.5 \mathrm{~dB}$.

The results also have shown that, in the absence of CCI, with SMR $=0 \mathrm{~dB}$ and $7 \mathrm{~dB}$, 2-branch diversity PSA-16QAM starts gaining advantages in the SNR at the BERs of $8 \times 10^{-3}$ and $4 \times 10^{-4}$, respectively, over non diversity QPSK. While in the absence of noise, with SMR $=0 \mathrm{~dB}$ and $7 \mathrm{~dB}$, 2-branch diversity PSA-16QAM starts gaining the advantages in SIRs at the BERs of $2 \times 10^{-3}$ and $5 \times 10^{-5}$, respectively, over non diversity QPSK. Thus at low error rates, PSA-16QAM with 2-branch diversity reception is superior to non diversity QPSK and so seems to be more preferred. However, in the CCI environments and in the absence of noise, 4-branch diversity QPSK outperforms 2-branch diversity PSA-16QAM in all the condition tested. 


\section{REFERENCES}

[1] K. M. S. Murthy, V. K. Bhargava, and A. Boettcher, "Personal SATCOM Systems and Services," Tutorial Record in Proceedings of the IEEE International Conference on Universal Personal Communications, San Diego, U.S.A., September 1994.

[2] D. C. Cox, "Wireless Personal Communications: What is it?," IEEE Personal Communications, vol. 2, no. 2., April 1995.

[3] W. T. Webb, "QAM: The Modulation Scheme for Future Mobile Radio Communications ?,” Electronics and Communication Engineering Journal, vol. 4, no. 4., 1992, pp. 167-176.

[4] H. K. Lau and S. W. Cheung, “A Fade-Compensation Technique for Digital Land Mobile Satellite Systems,” accepted, to appear in International Journal of Satellite Communications.

[5] J. K. Cavers, “An Analysis of Pilot Symbol Assisted Modulation for Rayleigh Fading Channels,” IEEE Transactions on Vehicular Technology, vol. 40, no. 4, pp. 686-693, November 1991.

[6] S. Sampei and T. Sunaga, "Rayleigh Fading Compensation for QAM in Land Mobile Radio Communications,” IEEE Transactions on Vehicular Technology, vol. 42, no. 2, pp. 137-147, May 1993.

[7] C. L. Liu and K. Feher, "Pilot-Symbol Aided Coherent $M$-ary PSK in FrequencySelective Fast Rayleigh Fading Channels," IEEE Transactions on Communications, vol. 42, no. 1, January 1994, pp. 54-62.

[8] H. K. Lau and S. W. Cheung, "A Pilot Symbol-Aided Technique Used for Digital Signals in Multipath Environments," in Proceedings of the IEEE International Conference on Communications, New Orleans, U.S.A., May 1994, pp. 1126-1130.

[9] P. Ho and J. H. Kim, "Pilot Symbol-Assisted Detection of CPM Schemes Operating in Fast Fading Channels," IEEE Transactions on Communications, vol. 44, no. 3, pp. 337-347, Mar. 1996.

[10] H. K. Lau and S. W. Cheung, "Performance of a Pilot Symbol-Aided Technique in Frequency-Selective Rayleigh Fading Channels Corrupted by Co-Channel Interference and Gaussian Noise," in Proc. of IEEE Veh. Technol. Conf., Atlanta, USA, April 1996, pp. 1008-1012.

[11] W. C. Jakes, Microwave Mobile Communications, New York, John Wiley \& Sons, 1974.

[12] F. Adachi and J. D. Parsons, "Error Rate Performance of Digital FM Mobile Radio with Predetection Diversity,” IEEE Transactions on Communications, vol. 37, no. 3, pp. 200-210, Mar. 1989.

[13] F. Adachi and K. Ohno, "BER Performance of QDPSK with Postdetection Selection Diversity Reception in Mobile Radio Channels,” IEEE Trans. Veh. Technol., vol. 40, no. 1, pp. 237-249, Feb. 1991.

[14] T. Eng, N. Kong, and L. B. Milstein, "Comparison of Diversity Combining Techniques for Rayleigh-Fading Channels," IEEE Transactions on Communications, vol. 44, no. 9, pp. 1117-1129, Sept. 1996.

[15] Inmarsat, INMARSAT Standard-C System Definition Manual, Module 4, 1989. 
[16] L. J. Mason, "Error Probability Evaluation for Systems Employing Differential Detection in a Rician Fast Fading Environment and Gaussian Noise," IEEE Transactions on Communications, vol. 35, no. 1, January 1987, pp. 39-46.

[17] H. Salwch, "Differential phase-shift keying performance under time-selecting multipath fading”, IEEE Transactions on Communications, vol. 23, pp. 383-385, 1975.

[18] W. H. Tranter and K. L. Kosbar, "Simulation of Communication Systems,” IEEE Communications Magazine, vol. 32, no. 7, pp. 26-35, July 1994.

[19] S. W. Cheung, “Digital Modems for Mobile Systems,” Ph.D. Thesis, Loughborough University of Technology, 1986.

[20] M. C. Jeruchim, P. Balaban, and K. S. Shanmugan, Simulation of Communication Systems, Plenum Press, New York, 1992. 
TABLE 1. SNRs for $N$-branch PSA-16QAM and QPSK to achieve the BERs of $10^{-2}, 10^{-3}$, $10^{-4}$ and $10^{-5}$

\begin{tabular}{|c|c|c|c|c|c|c|}
\hline \multirow[t]{2}{*}{ BER } & \multicolumn{4}{|c|}{$\begin{array}{c}\text { PSA-16QAM } \\
\text { dB }\end{array}$} & \multicolumn{2}{|c|}{$\begin{array}{c}\text { QPSK } \\
\mathrm{dB}\end{array}$} \\
\hline & $N=1$ & $N=2$ & $N=3$ & $N=4$ & $N=1$ & $N=2$ \\
\hline \multicolumn{7}{|c|}{ (a) $\mathrm{SMR}=0 \mathrm{~dB}$} \\
\hline $10^{-2}$ & 16 & 10 & 8 & 7 & 9.5 & 3 \\
\hline $10^{-3}$ & 30 & 16 & 12.5 & 11 & 19.5 & 8.5 \\
\hline $10^{-4}$ & - & 21.5 & 16.5 & 14.5 & 28.5 & 13 \\
\hline $10^{-5}$ & - & - & 20.5 & 17.5 & - & 19 \\
\hline \multicolumn{7}{|c|}{ (b) $\mathrm{SMR}=7 \mathrm{~dB}$} \\
\hline $10^{-2}$ & 13 & 10.5 & 9.5 & 9 & 6.5 & 3.5 \\
\hline $10^{-3}$ & 19 & 14.5 & 13 & 12.5 & 13 & 7 \\
\hline $10^{-4}$ & 27.5 & 18 & 16 & 15.5 & 20 & 10 \\
\hline $10^{-5}$ & - & 20.5 & 18.5 & 17.5 & 29 & 12.5 \\
\hline
\end{tabular}

TABLE 2. SIRs for $N$-branch PSA-16QAM and QPSK to achieve the BERs of $10^{-2}, 10^{-3}$, $10^{-4}$ and $10^{-5}$

\begin{tabular}{|c|c|c|c|c|c|c|}
\hline \multirow[t]{2}{*}{ BER } & \multicolumn{4}{|c|}{$\begin{array}{c}\text { PSA-16QAM } \\
\text { dB }\end{array}$} & \multicolumn{2}{|c|}{$\begin{array}{c}\text { QPSK } \\
\mathrm{dB}\end{array}$} \\
\hline & $N=1$ & $N=2$ & $N=3$ & $N=4$ & $N=1$ & $N=2$ \\
\hline \multicolumn{7}{|c|}{ (a) $\mathrm{SMR}=0 \mathrm{~dB}$} \\
\hline $10^{-2}$ & 21.5 & 15.5 & 14 & 13 & 13 & 6.5 \\
\hline $10^{-3}$ & - & 21.5 & 19.5 & 18 & 22.5 & 12 \\
\hline $10^{-4}$ & - & 28.5 & 23.5 & 22 & - & 17 \\
\hline $10^{-5}$ & - & - & 28 & 25.5 & - & 21.5 \\
\hline \multicolumn{7}{|c|}{ (b) $\mathrm{SMR}=7 \mathrm{~dB}$} \\
\hline $10^{-2}$ & 19 & 16.5 & 16 & 16 & 9 & 7 \\
\hline $10^{-3}$ & 25.5 & 21.5 & 20.5 & 20 & 16 & 10.5 \\
\hline $10^{-4}$ & - & 25 & 24 & 23 & 23 & 13 \\
\hline $10^{-5}$ & - & 28 & 26.5 & 26 & - & 16 \\
\hline
\end{tabular}

\section{LIST OF FIGURES}


Fig. 1 Baseband Equivalent Model of System

Fig. 2 Frame Structure of Transmitted Symbol Sequence

Fig. 3 (a) Model of Transmission Path,

(b) Model to Generate Multipath Component

Fig. 4 BER Performances as function of SNR, with SIR $=\infty \mathrm{dB}$ at (a) $\mathrm{SMR}=0 \mathrm{~dB}$, and (b) $\mathrm{SMR}=7 \mathrm{~dB}$

Fig. 5 BER Performances as function of SIR, with $S N R=\infty \mathrm{dB}$ at (a) $\mathrm{SMR}=0 \mathrm{~dB}$, and (b) SMR $=7 \mathrm{~dB}$ 


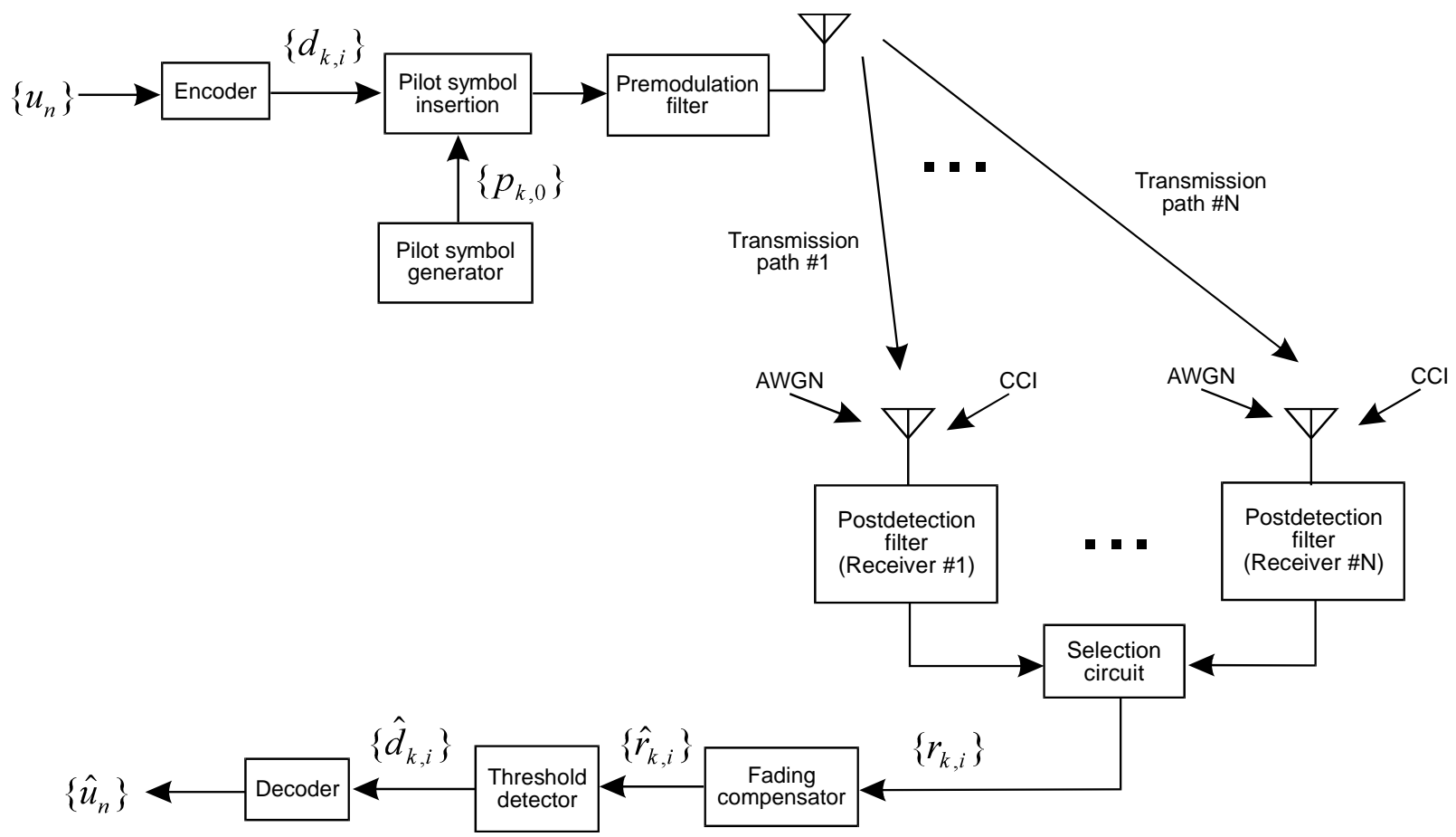

Fig. 1 Baseband Equivalent Model of System

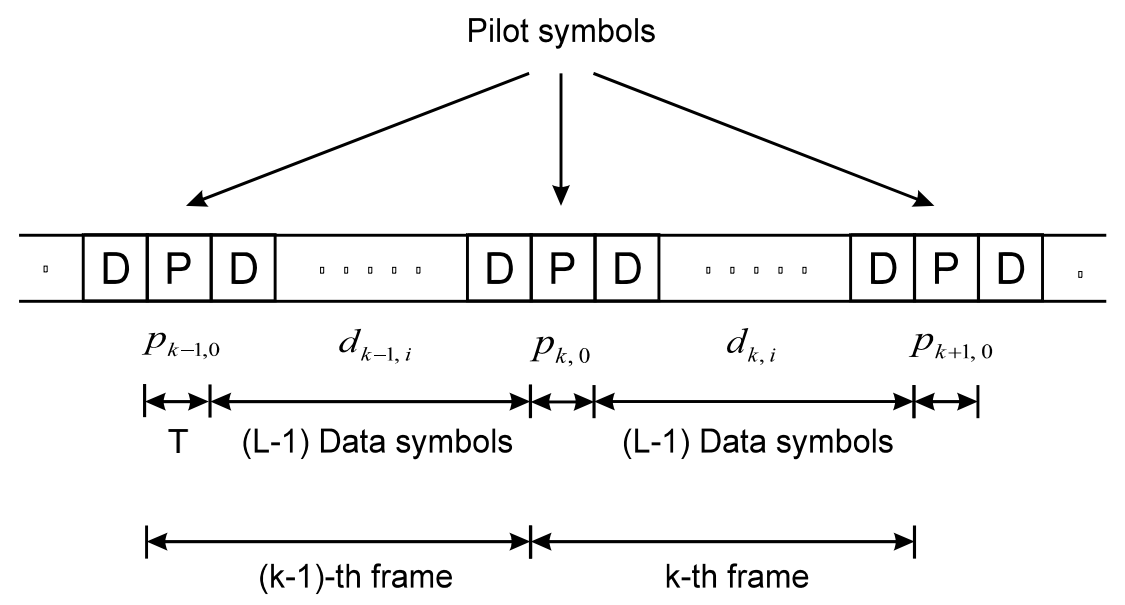

Fig. 2 Frame Structure of Transmitted Symbol Sequence 


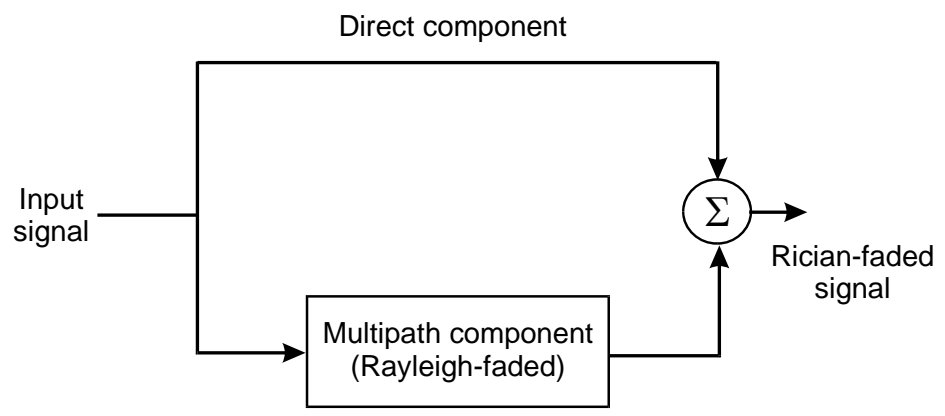

Fig. 3a Model of Transmission Path

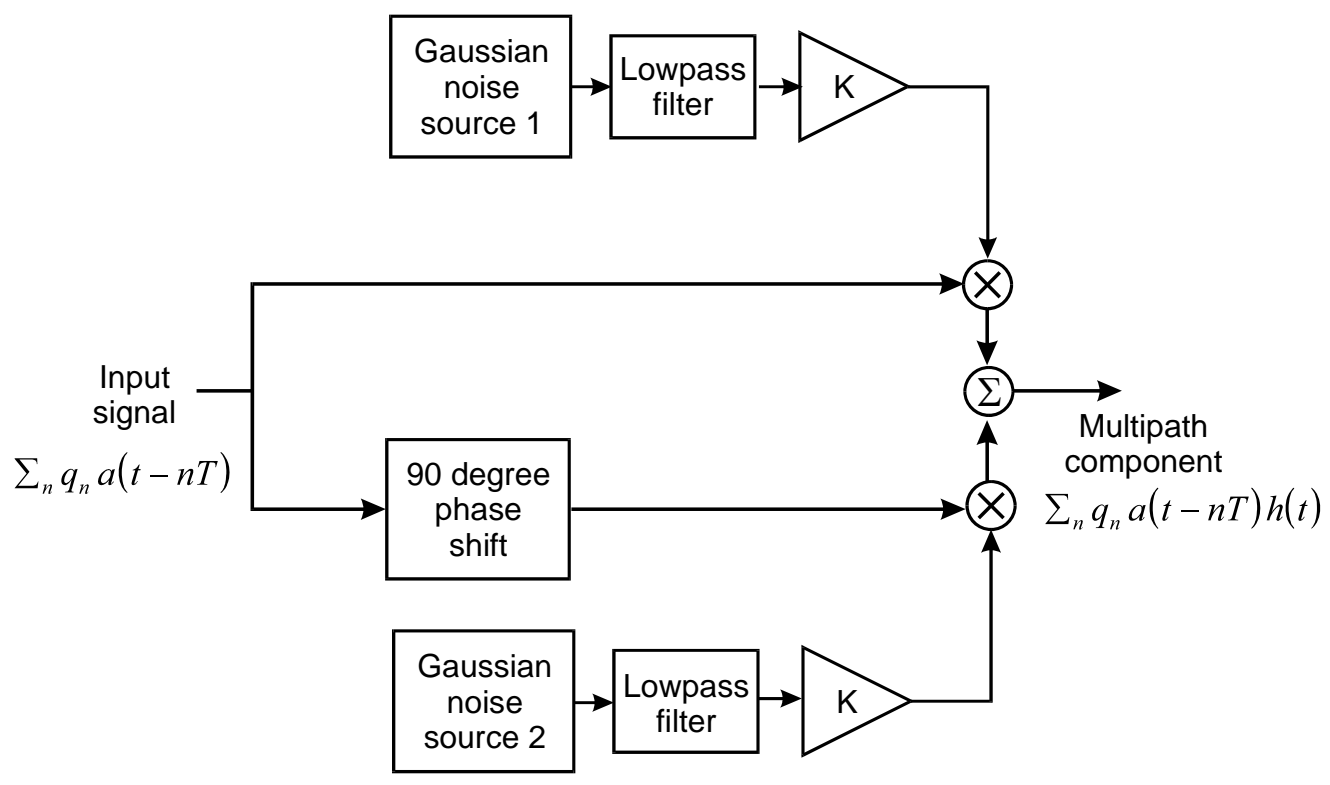

Fig. 3b Model to Generate Multipath Component 


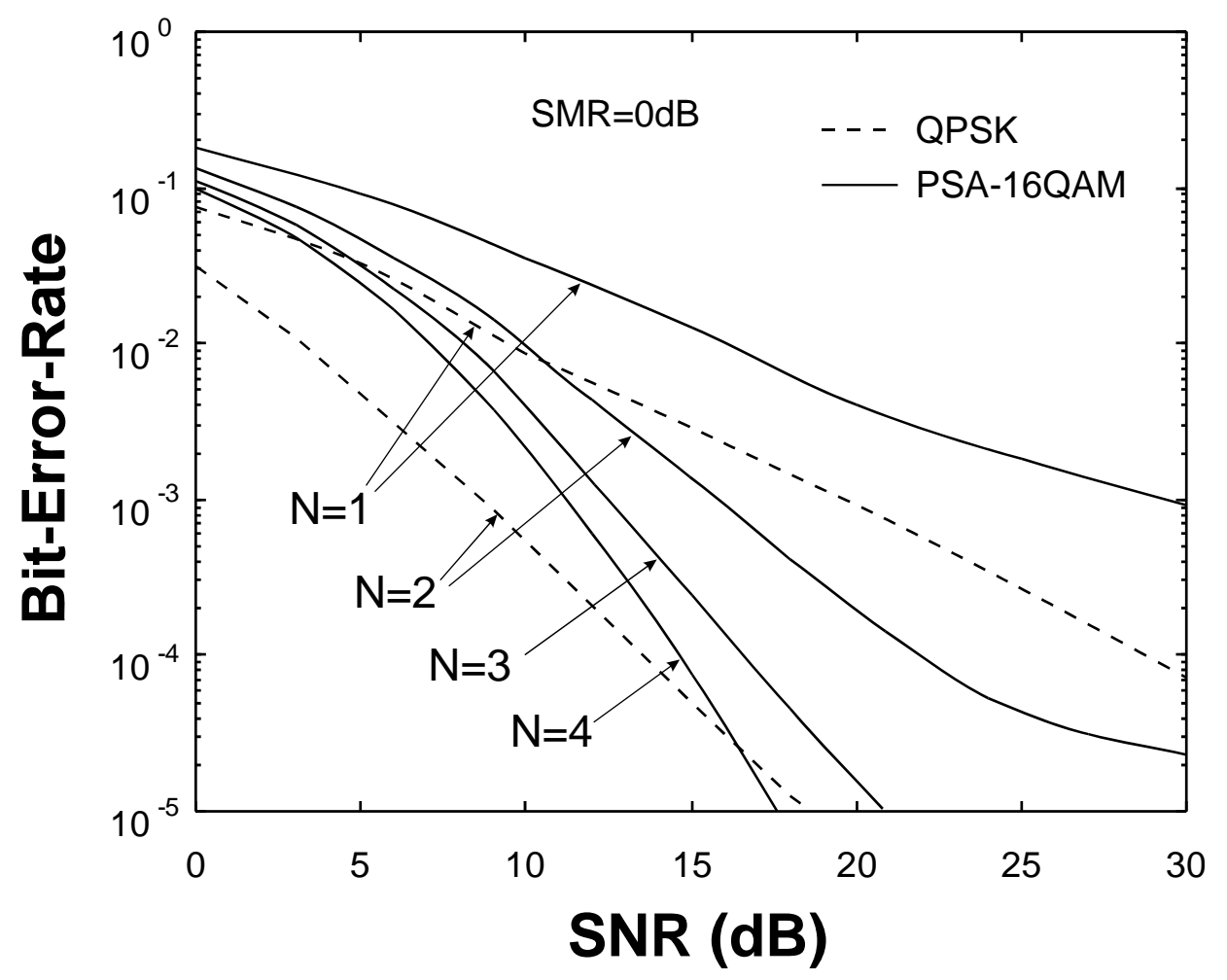

Figure 4a. BER performance as function of SNR, with SIR $=\infty \mathrm{dB}$ and $\mathrm{SMR}=0 \mathrm{~dB}$

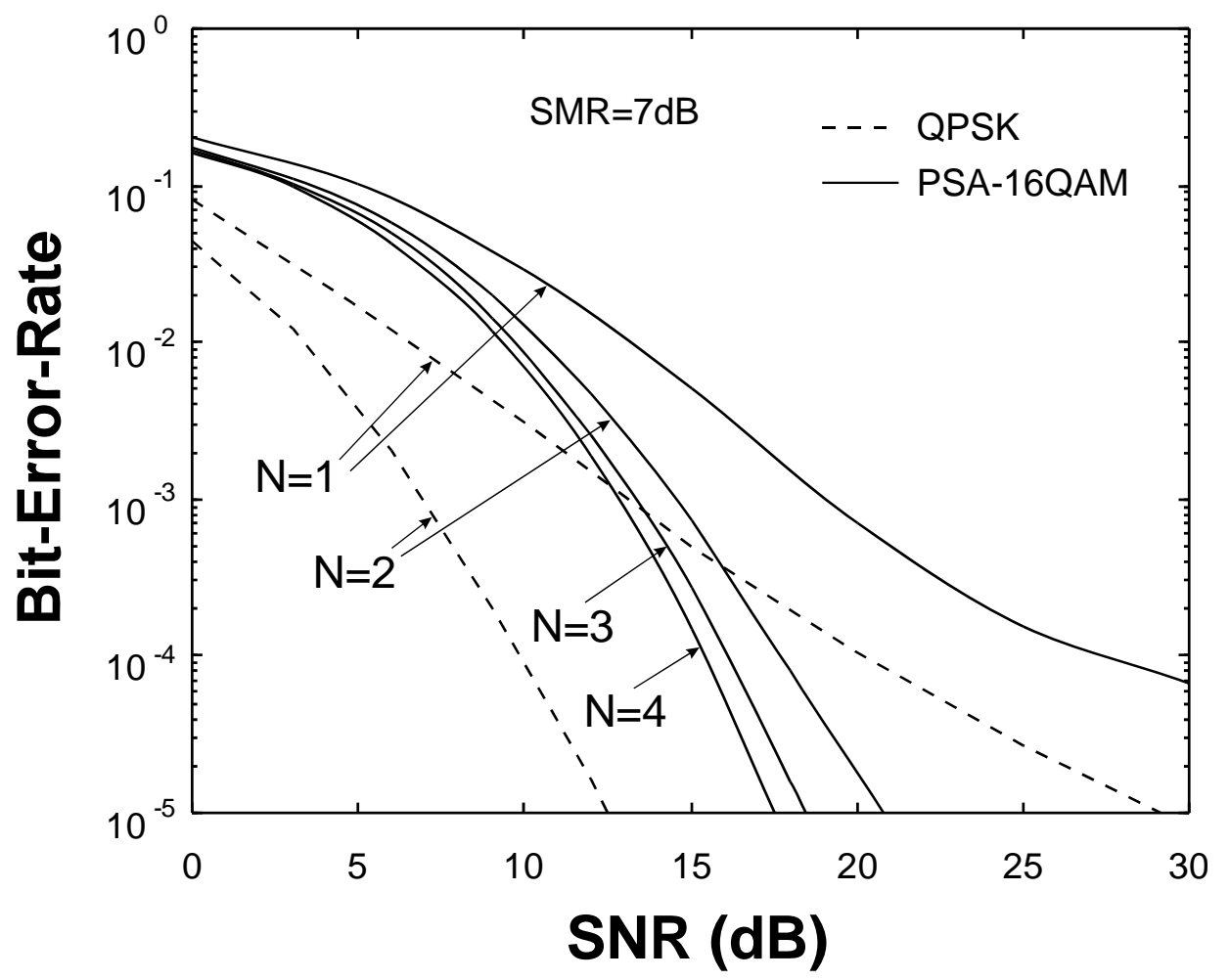

Figure $4 \mathrm{~b}$. BER performance as function of $\mathrm{SNR}$, with $\mathrm{SIR}=\infty \mathrm{dB}$ and $\mathrm{SMR}=7 \mathrm{~dB}$ 


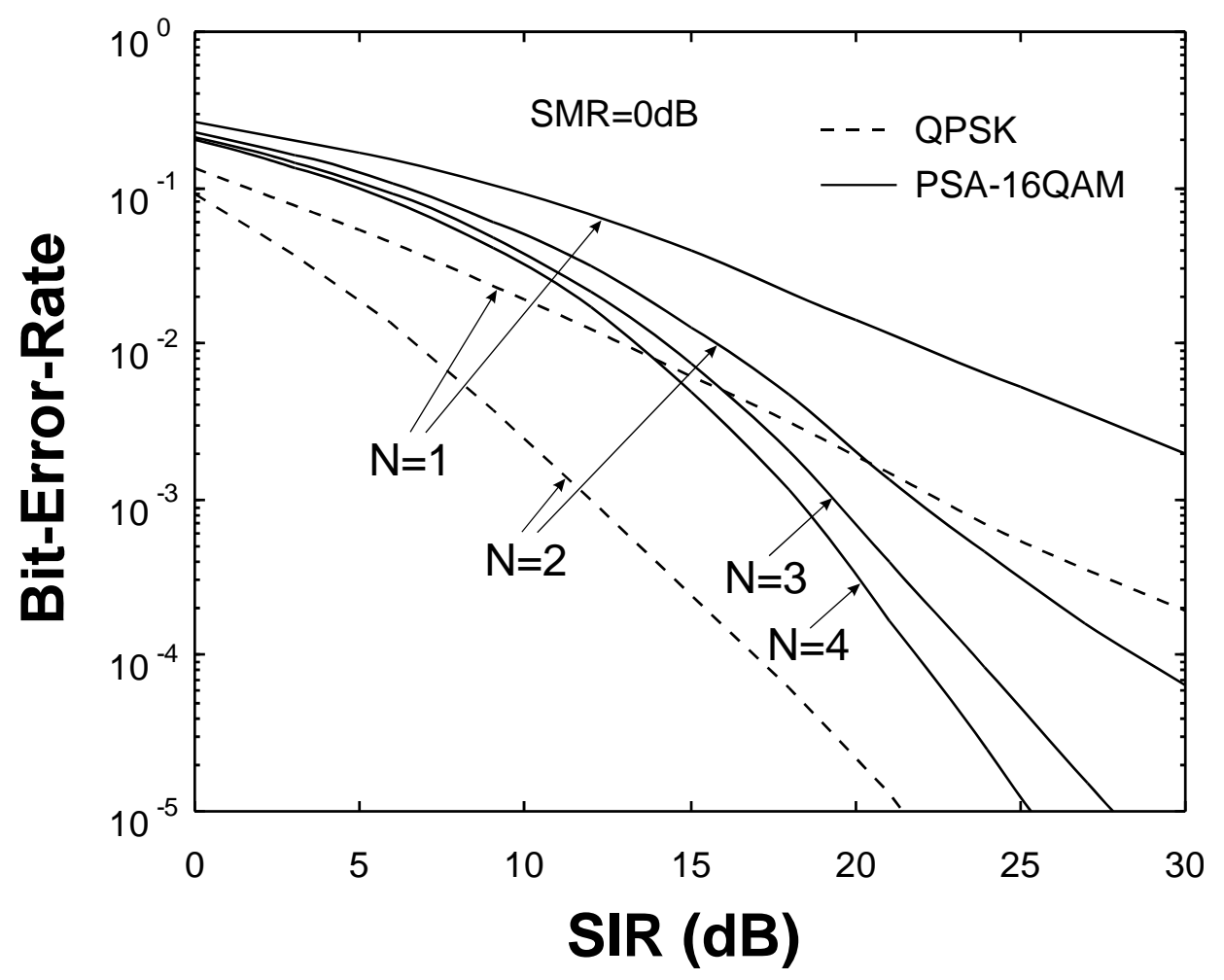

Figure 5a. BER performance as function of SIR, with SNR $=\infty \mathrm{dB}$ and SMR $=0 \mathrm{~dB}$

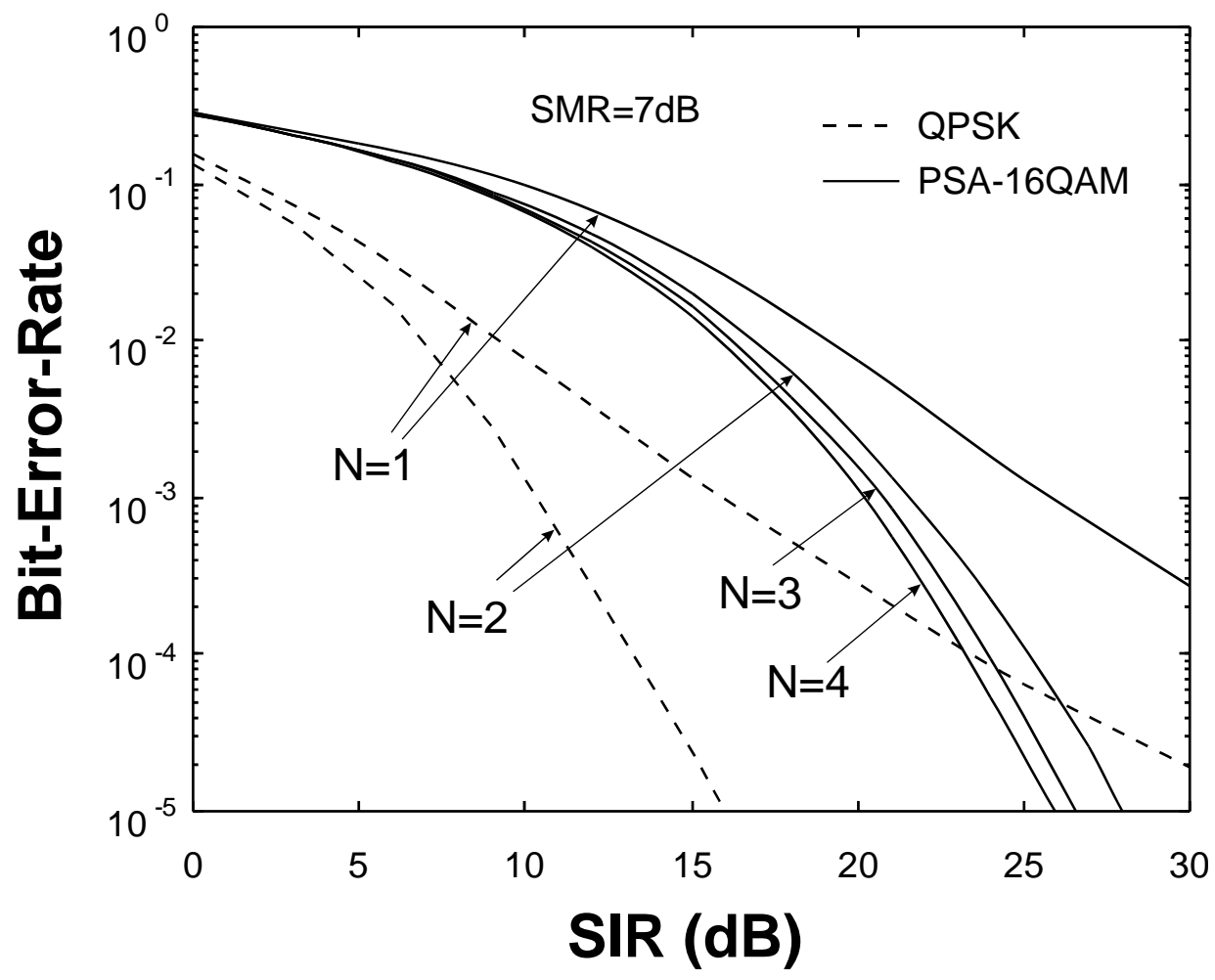

Figure 5b. BER performance as function of SIR, with SNR $=\infty \mathrm{dB}$ and SMR $=7 \mathrm{~dB}$ 